\title{
ENHANCED SEAT BELT REMINDER SYSTEMS FOR TEENAGE DRIVERS AND PASSENGERS
}

\author{
Neil Lerner, Jeremiah Singer \& Mark Freedman \\ Westat \\ Rockville, MD, USA \\ Email: neillerner@westat.com
}

\begin{abstract}
Summary: Failure to use a seat belt is a significant highway safety concern for teenagers. The current Federally-required seat belt reminder system is limited in its effectiveness, and many automobile manufacturers are now providing enhanced seat belt reminder (ESBR) systems. Current systems are designed for the general driving public and their design must represent a trade-off between effectiveness in promoting belt use and consumer acceptance. Teens may respond differently to system features and trade-off considerations may be different for risk-prone teens. This study conducted research to evaluate teen driver and passenger reactions to a variety of ESBR systems and features. The study was conducted in an operational, but stationary vehicle, with simulated drives. Systems and features were evaluated regarding their likelihood of increasing belt use, annoyance, signal appropriateness, desirability, and other aspects. Discussion groups were also held with the parents of teen drivers. Based on findings of the experiment and discussions, a set of recommendations was developed for the design of optimal ESBR systems oriented toward teen drivers and their passengers.
\end{abstract}

\section{INTRODUCTION}

Teen drivers and passengers are particularly in need of improved rates of seat belt use. Their overrepresentation in vehicle crashes and fatalities alone would justify this. But beyond that, teens have lower seat belt use rates than the adult driving population and failure to use a seat belt is typical of teen crash fatalities. In 2006, only 39\% of 16-19 year old drivers killed in crashes were belted and only 27\% of 16-19 year old passengers killed were belted (Traffic Safety Facts: Young Drivers, 2008).

Enhanced seat belt reminder (ESBR) systems are one approach to improve seat belt use. The current Federally-required reminder system (under FMVSS 208) requires an audible signal of 4 to 8 seconds duration when the ignition is turned on and a warning light for no less than one minute if the driver's seat belt is not buckled. This minimum requirement appears limited in its effectiveness for drivers (Transportation Research Board, 2003) and does not address passenger seat belt use at all. Many automobile manufacturers are now voluntarily installing ESBR systems in new vehicle models. ESBRs typically extend and/or supplement the basic visual and auditory reminders, but also may include other features. ESBRs increase the rate of driver and passenger belt use and the literature provides some indication of desirable ESBR features (Freedman, Lerner, Zador, Singer, \& Levi, 2008; Kraft, Kullgren, Lie, \& Tingvall, 2006; Williams, Wells, \& Farmer, 2002). Current products, prototype systems, and systems recommended in the literature (e.g., Eby, Molnar, Kostyniuk, \& Shope, 2004) vary widely in their attributes. 
ESBRs can improve the use of seat belts in two ways: they may remind forgetful vehicle occupants to buckle their seat belts and they may motivate reluctant occupants to buckle their seat belts in order to avoid or terminate undesired signals. ESBR systems represent a compromise between potential effectiveness and consumer acceptability. For example, an extremely loud and harsh sound immediately upon engine ignition might get most people to buckle up immediately, but simply would not be acceptable as a consumer product. Many ESBR systems have a delay before the reminder phase and many are staged so that signals are initially moderate and then more assertive.

Optimal design decisions and trade-offs may not be the same for teen drivers and passengers compared to the general driving public. Teens may respond differently to system features and trade-off decisions may be different for risk-prone teens. Some research has demonstrated that ESBRs can increase the rate of seat belt use for teens but little indicates what sorts of features are most effective and appropriate for teen drivers and their passengers. Given the particular problem of seat belt nonuse among teens, there is a need to design an effective teen-oriented ESBR system. This paper describes a research project to investigate ESBRs for teens and develop recommendations for teen-oriented systems.

This work inherently presumes that the vehicle system can recognize when the driver is a teen. This is currently feasible through various technologies, such as smart keys, biometric recognition, or selectable programming of vehicle options by the parent. For example, Ford has announced the MyKey technology which will debut as standard equipment on the 2010 Ford Focus. It allows vehicle owners (parents) to program certain limits and features, including more aggressive seat belt reminder functions. While teen driver recognition is an assumption of this project, driver recognition technologies themselves were not the subject of this research.

\section{OBJECTIVE AND SCOPE}

The present study was part of a larger effort to evaluate the effectiveness of ESBR systems. Two earlier phases of the project were a field observational study of belt use in vehicles with known types of ESBR systems (Freedman, Levi, Zador, Lopdell, \& Bergeron, 2007) and an in-vehicle experiment to investigate the acceptability and potential effectiveness of ESBR features for adult driver populations (Lerner, Singer, Huey, \& Jenness, 2007). The present study addressed how ESBR system characteristics related to teen driver and passenger responses regarding the likelihood of wearing a seat belt, annoyance, signal appropriateness, desirability, and other aspects. Data were collected in a functional, but stationary, vehicle with simulated drives. Demonstration/discussion groups were also held with the parents of teen drivers. The project developed a set of design recommendations for optimal ESBR systems oriented toward teens.

\section{METHOD}

The method of this study involved multiple procedures and a range of vehicle displays, ESBR algorithms, and dependent measures. Due to the restricted length of this paper, only an overview of the method can be provided. A detailed description of the instrumentation, displays, and methods will be available in the project final report (Lerner, Singer, Freedman, Robinson, Huey, \& Walrath, in preparation). 
The study included 103 teen drivers and passengers, ages 16 to 18, who were not consistent seat belt users. Thiry-one participants were tested as solo drivers, alone in the test vehicle. The other 72 partipicants were tested in "affinity groups" of three or four friends present in the vehicle together. The affinity groups allowed evaluation of ESBRs for drivers, front seat passengers, and rear seat passengers and also promoted normal teen peer social interaction (ecouraged by instruction). The experiment was conducted in a functional vehicle (2001 Saturn L200 sedan) that was modified so that a variety of custom ESBR displays (visual, acoustic, and haptic seat pan vibration) and timing algorithms could be implemented under experimenter control. For this experiment, the vehicle was stationary. Roadway drives were simulated with a driver's view video of the road projected on a screen directly in front of the vehicle. The driver was engaged in a steering wheel tracking task (keeping a cursor in the center of the roadway), in order to promote out-the-windshield attention, encourage normal driving postures, and prevent the driver from focusing attention on ESBR displays. A string potentiometer attached to the steering column adjusted the cursor position in the video in real time.

Different procedures were used for solo and group sessions. In both procedures, participants first experienced seat belt reminder systems while engaging in 3-minute video driving simulations and provided feedback about the systems. Solo participants were unbelted for all simulated drives (and therefore experienced all ESBR features) while group participants were unbuckled for some drives and unbuckled for others. Mixed seat belt use allowed the evaluation of group social dynamics and participants' willingness to encourage their friends to buckle up. At least one group participant was unbuckled for each simulated drive. Periodically during each simulated drive, participants rated the likelihood that they would buckle up (or encourage fellow occupants to buckle up, for participants who were belted), how annoyed they were by the seat belt reminder, and the appropriateness of the strength of the alerts. At the completion of each drive, they answered additional questions about the system.

Participants in the group sessions experienced drives with the current minimal seat belt reminder system and six distinct ESBR systems devised to include different types of displays and strategies. A total of 12 trips were included, where some systems were repeated with different combinations of belted and unbelted vehicle occupants. Following the video drives, the group of teens took part in a brief discussion period moderated by the experimenter.

Participants in the solo sessions experienced simulated trips with the minimal system and five of the ESBR systems, for a total of six drives. Following the simulated driving portion of the study, participants in solo sessions experienced the initial "reminder" phase (about 30 seconds) for 12 distinct systems. These were displayed without the video simulation and participants rated the likelihood that they would buckle up, how annoyed they were by the seat belt reminder, and the appropriateness of the strength of the alerts. The final portion of the solo sessions involved the presentation of 27 brief individual alerts (about 6 seconds in duration), including various visual icon displays, text displays, auditory displays, voice messages, seat pan vibration, and combinations of these. The final alert for each participant was a trial with the radio on for which a sound system lockout was triggered. For each of the 27 alerts, the participant rated the likelihood of buckling up in response to the signal and the annoyance of the signal. 
Demonstration/discussion sessions were also held with groups of three or four parents of teen drivers, with a total of 25 parents. The various ESBR systems and display elements used in the teen experiment were demonstrated to the parents in the same test vehicle. During the demonstration, they rated how they thought the system would affect their own teen's belt use, belt use by teens who rarely wear seat belts, and the overall desirability of the system. Following this, there was an experimenter-moderated group discussion, which included parents' opinions about teen seat belt use, ESBR preferences, teen preferences, ESBR customization, value of ESBRs, and methods to educate parents and teens about ESBR options.

\section{FINDINGS AND TEEN-ORIENTED ESBR SYSTEM DESIGN RECOMMENDATIONS}

To permit presentation of the recommendations for teen-oriented ESBR design, only a few selected findings are presented here for illustrative purposes. The design of the teen experiment was complex and involved numerous variables for each of the three distinct procedures, including occupant gender, seat position, belt use status, social setting (solo, all male, all female, mixed gender), display mode, display features, algorithm timing, and more. Full treatment of findings will be available in Lerner et al. (in preparation). Figure 1 below shows teens' postdrive ratings of ESBR effectiveness in encouraging them to buckle their seat belts. The data show that all ESBRs were significantly more effective than the minimal seat belt reminder required by law. The ESBR systems themselves showed a range of effectiveness, and there were also effects of the social setting (solo, peer group) and seat position. Figure 2 shows teens' mean ratings of each of the 27 individual alerts, showing the mean rating of effectiveness and annoyance for each alert. The displays spanned a broad range of effectiveness and annoyance. The figure shows that perceived effectiveness and annoyance of alerts were strongly correlated, and few alerts deviated substantially from this correlative pattern. The project report explains the features of the systems and alerts shown in these figures and relates dependent measures to the range of display attributes and other experimental conditions.

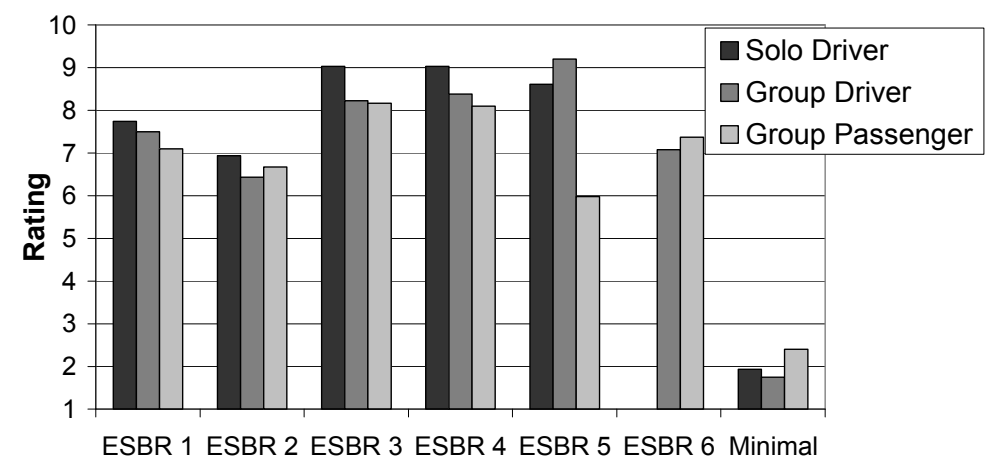

Figure 1. Teens' ratings of ESBR effectiveness 


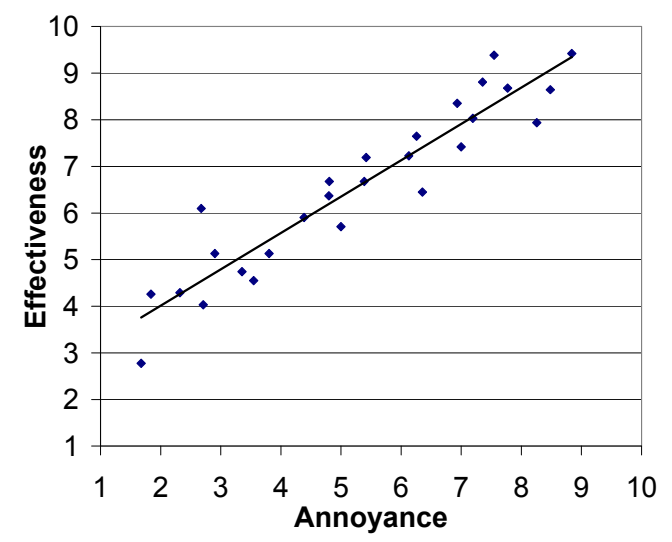

Figure 2. Solo participants' mean ratings of individual alerts: Perceived effectiveness versus annoyance

\section{Development of Design Recommendations}

The findings of the teen ESBR experiment and the parent discussion groups, together with the findings of the complementary observational and experimental studies in this project (Freedman et al., 2008) and other findings in published research literature were used to generate a set of recommendations for the design of teen-oriented ESBR systems. The set of recommendations is hierarchical, in that a particular general recommendation was followed by more specific recommendations on sub-aspects. For each recommendation, a specific rationale statement provides the empirical and logical basis for the details of the recommendation. The set of recommendation statements is provided below. Due to the space limitations of this paper, the rationale statements are not included. The rationales are important for understanding why a particular recommendation is desirable, which may not always be evident from the recommendation statement itself. A full treatment of the recommendations, with complete rationale, will be available in the project final report (Lerner et al., in preparation).

\section{Recommendations for Teen-Oriented ESBR Systems}

Driver specificity. The ESBR should be able to determine when the vehicle is operated by a novice teen driver.

Graded alerts. The enhanced display should include two phases. The "reminder" phase should be an effective, but not highly annoying reminder. The subsequent "motivator" phase should be more assertive and motivate belt use.

- Criteria for initiating phases of the enhanced reminder system: The initial (reminder) phase should occur about 8 seconds following engine ignition, assuming the vehicle is stationary. The more assertive motivator phase should start no more than 15-20 seconds after engine ignition or as soon as the vehicle exceeds a minimum speed threshold, whichever comes first. The minimum speed threshold should be that at which sustained motion can be reliably confirmed.

- Subsequent to an initial cycle of the motivator phase, the vehicle should be in motion for the algorithm to continue. Timing should be suspended while the vehicle is stationary and not in forward or reverse gear.

- Alert levels should not be speed dependent. 
- The motivator phase should cycle at a frequency of at least once every 30 seconds (a cycle length or period of at most every 30 seconds), with at least 5 seconds of auditory signal, or a voice message, per cycle.

- The motivator phase of the reminder system should continue for as long as the teen driver is unbelted.

Modality. Visual displays are necessary but not sufficient. Acoustic signals (sounds or speech) are essential, particularly for the motivator phase. Tactile displays may be useful supplements. At this point, it is not known whether tactile stimuli are sufficient to replace acoustic signals.

- Acoustic displays should take into account the following research findings:

- Acoustic signals are more effective with faster repetition rates and greater sound level. Effectiveness is highly correlated with annoyance.

- Voice messages appear comparable in effectiveness, but somewhat less annoying, than other auditory signals (chimes, tones). They were positively received by teens, and the parents of teens also liked the voice option, finding it unambiguous, authoritative, and unlikely to be confused with other sounds that may be present in the vehicle. Voice messages should be given strong consideration for teen-oriented reminder systems. They must be intelligible in the operational environment, which may include multiple teens and infotainment system use at loud levels.

- Acoustic signals for seat belt reminders should be distinct from and not confusable with other in-vehicle alerts.

- Visual displays should take into account the following research findings:

- Flashing displays are more attention-getting and motivating than steady displays

- Flashing displays are less motivating than all but the least assertive acoustic signals, but are also less annoying

- Center console screens, by virtue of their size and location, are a particularly effective means of presenting visual displays and are seen as desirable by teen drivers.

- Tactile displays should take into account the following research findings:

- Teens rated tactile displays relatively highly for effectiveness and desirability, particularly as used in conjunction with speech messages. The tactile signal used in the research was seat pan vibration; other tactile signals could be considered.

- Teens and parents both expressed concern that seat vibration could startle teen drivers. Data are lacking on this issue, but if tactile displays are used, design consideration should be given to potential startle reactions by drivers, particularly inexperienced ones.

Passenger status and display. A teen-oriented ESBR system should sense the belt use status of passengers in all seat positions. The system should be designed to directly encourage unbelted occupants to buckle up and to encourage belted occupants to speak up to unbelted occupants.

- Unbelted passengers should receive a sustained flashing visual display.

- Passenger seat belt status should be visually displayed to the driver.

- The presence of any unbelted occupant should provide some periodic signal that is perceptible to all occupants. 
Vehicle adaptation. If a teen driver is not belted, aspects of vehicle performance may be modified, through lockouts or limiters.

- An indicator display should be continuously presented if a vehicle feature has been locked out or limited. The display should indicate both the feature that has been altered and that seat belt use is required to restore the function.

Customization. There may be a driver-selectable or adult-selectable component to the reminder phase of the ESBR system, but not the features of the subsequent motivator phase.

\section{CONCLUSIONS}

The responses of both teen drivers and teen passengers are sensitive to the display features and timing algorithms of ESBR systems. The findings of this study suggest a variety of design factors that may contribute to highly effective teen-oriented ESBR systems. These recommendations differ somewhat from what may be optimal or acceptable in ESBR systems designed for the general driving public. Assuming that vehicle systems are able to recognize when a teen is operating the vehicle, systems can be put in place that are effective, desirable to teen's parents, and acceptable as consumer products. Given the current interests of automobile manufacturers in ESBR systems, together with recognition of the serious teen crash risk problem, it is hoped that these guidelines will promote alternative ESBR system designs that are especially effective in getting at-risk teen drivers and passengers to more reliably wear seat belts.

\section{ACKNOWLEDGMENTS}

This work was conducted under funding from the National Highway Traffic Safety Administration. The authors thank Jennifer Warren and Stephanie Binder of NHTSA for their support and contributions throughout the project.

\section{REFERENCES}

Eby, D., Molnar, L., Kostyniuk, L., \& Shope, J. (2004). Developing an optimal in-vehicle safety belt promotion program (Report no. UMTRI 2004-29). Ann Arbor, Michigan: University of Michigan Transportation Research Institute.

Freedman, M., Lerner, N., Zador, P., Singer, J., \& Levi, S. (2008). Effectiveness and acceptance of enhanced seat belt reminder systems: Characteristics of optimal reminder systems. Report prepared by Westat and submitted to National Highway Traffic Safety Administration.

Freedman, M., Levi, S., Zador, P., Lopdell, J., \& Bergeron, E. (2007). The Effectiveness of enhanced seat belt reminder systems: Observational field data collection methodology and findings. Report no. DOT HS 810 844. Washington, DC: National Highway Traffic Safety Administration.

Krafft, M., Kullgren, A., Lie, A., \& Tingvall, C. (2006). The use of seat belts in cars with smart seat belt reminders: Results of an observational study. Traffic Injury Prevention, 7(2), 125129. 
Lerner, N., Singer, J., Huey, R., \& Jenness, J. (2007). Acceptability and potential effectiveness of enhanced seat belt reminder system features. Report no. DOT HS 810 848. Washington, DC: National Highway Traffic Safety Administration.

Lerner, N., Singer, J., Freedman, M., Robinson, A.E, Huey, R., \& Walrath, J. (in preparation). Acceptability and potential effectiveness of enhanced seat belt reminder system features: teen drivers and parents of teens. Report in preparation under National Highway Traffic Safety Administration contract DTNH22-5-D-01002, Task Order 1.

Traffic safety facts: 2006 data: Young drivers (2008). Report no. DOT HS 810 817. Washington, DC: National Highway Traffic Safety Administration.

Transportation Research Board (2003). Buckling up: Technologies to increase seat belt use (TRB Special Report 278). Washington, DC: Transportation Research Board.

Williams, A., Wells, J., \& Farmer, C. (2002). Effectiveness of Ford's enhanced belt reminder system in increasing seat belt use. Injury Prevention, 8, 293-296. 